\title{
The effect of SIRT1 protein knock down on PGC-1 $\alpha$ acetylation during skeletal muscle contraction
}

\author{
Dae Ryoung Park', Jeong Seok Kim ${ }^{1}$ and Chang Keun Kim ${ }^{2 *}$ \\ ${ }^{1}$ Department of physical education, Chonbuk National University, Jeonju, Korea \\ ${ }^{2}$ Human Physiology, Korea National Sport University, Seoul, Korea
}

(Received: 2014/01/02, Revised: 2014/02/04, Published online: 2014/02/11)

[Purpose] The purpose of this study was to investigate the effect of Sirtuin 1 (SIRT1) and General control nonderepressible 5 (GCN5) knock down on peroxisome proliferator- activated receptor gamma coactivator 1-alpha (PGC-1a) deacetylation during electrical stimulated skeletal muscle contraction. [Methods] Skeletal muscle primary cell were isolated from C57BL/6 mice gastrocnemius and transfected lentiviral SIRT1 and GCN5 shRNA. Knock downed muscle cell were stimulated by electrical stimulation (1Hz, 3min) and collected for PGC-1a deceatylation assays. Immunoprecipitation performed for PGC-1a deacetylation, acetyl-lysine level was measured. [Results] Our resulted showed SIRT1 knock down not influenced to PGC-1a deacetylation during electrical stimulation induced muscle contraction while GCN5 knock down decreased PGC-1a deacetylation significantly $(p<0.05)$. [Conclusion] This study can be concluded that GCN5 is a critical factor for muscle contraction induced PGC-1a deacetylation. [Keyword] SIRT1, p-AMPK, p-ACC, GCN5, Deacetylation of PGC-1

\section{INTRODUCTION}

Regular exercise is widely recommended for preventing or management of metabolic diseases such as obesity, hypertension, coronary artery disease, type 2 diabetes. Many studies currently undergo to identify its effectiveness. Physical exercise or muscle contraction not only spends intercellular energy but also activates non-exercise thermogenesis through upregulation of energy metabolism related protein transcription in skeletal muscle which ameliorates metabolic diseases [1]. Sirtuin1 (SIRT 1) and peroxisome proliferator- activated receptor gamma coactivator 1-alpha (PGC-1a) are well known transcription factors which plays pivotal role in intercellular energy metabolism and gene regulation signal [2,3]. SIRT1 is a member of the sirtuin protein family which activated by chemical agonist such as resveratrol and metformin. SIRT1 activity is controlled by AMP/ATP and $\mathrm{NAD}^{+}$levels which regulated protein deacetylation in various cell [4]. During skeletal muscle contraction, AMP activated protein kinase (AMPK) and nicotinamide phosphoribosyltransferase (NAMPT) through salvage pathways caused increasement of intracellular
$\mathrm{NAD}^{+}$level. Increased $\mathrm{NAD}^{+}$stimulates SIRT1, a number of NAD cyclases, produces 2-O-acetyl-ADP ribose and deacetylates numerous transcription factors which involved in energy production such as PGC-1a. These process activates various proteins which involved in fatty acid oxidation or mitochondrial biogenesis [5].

Recently, several studies suggested possibility of novel AMPK-SIRT1-PGC-1a signal pathway during muscle contraction. In SIRT1 knock out (KO) mice, exercise induced energy production not influenced by SIRT1 KO in skeletal muscle [6], moreover overexpression of SIRT1 decreased PGC-1a expression [7]. Thus these result indicated that SIRT1 might be not versatile role in PGC-1a signal pathway during the skeletal muscle contraction however exact mechanism is not clear

PGC-1a is known to contain more than twelve of catalytic binding domains and regulates the gene transcription involved in energy metabolism. When PGC-1a is deacetylated by cyclic AMP (cAMP) signal pathway or cytokine signal pathway, it move into the nuclei which caused upregulation of uncoupling protein 1 (UCP1) that is known to activate mitochondrial

\footnotetext{
* Corresponding author: Chang-Keun Kim, Tel. 82-2-410-6815, Fax. 82-2-410-6769, Email. ckkim@knsu.ac.kr

(c)2014 The Korean Society for Exercise Nutrition
} 
energy metabolism which stimulate oxidation of fat in mitochondria [8]. PGC-1a normally remains acetylated form (unactivated) in cell, however when exercise or muscle contraction, SIRT1 deacetylates PGC-1a which changed to activated form of PGC-1a. Deacetylated PGC-1a moves into the nuclei to activate energy metabolism related gene transcription [9]. However, PGC-1a can be activated not only by SIRT1 but also by diverse factors such as steroid receptor coactivator-1 (SRC-1) and CREB-binding protein (CBP)/ P300 [10]. PGC-1a deacetylation also regulated by general control nonderepressible 5 (GCN5) and protein kinase a (PKA) which can induce non-exercise induced thermogenesis during recovery $[6,11]$.

Previous studies not provided exact mechanism for exercise induce SIRT1/PGC-1a deacetylation mechanism. More diverse in vivo or in vitro experiments using deletion of various transcription factors would be need. Therefore, the purpose of this study was to investigates the expression mechanism of PGC-1a deacetylation through knock down of GCN5 and SIRT1 genes, primary histone acetylation protein which involve in PGC-1a deacetylation during skeletal muscle cell contraction.

\section{METHODS}

\section{Experimental animal}

8-week-old C57BL/6 mice were used for this study. All mice were housed in plastic cages and were given standard feed and water. The temperature, humidity in the room were maintained at $23-25^{\circ} \mathrm{C}, 60-70 \%$, and Light was controlled by 12 hour cycle respectively. The mice weighed between 24 \pm 1.54 g. Management and experiment procedures for the animals used in this study cared in compliance with ethics regulations of Animal Testing Ethics Committee of Chonbuk University (2012-1-0039).

\section{Separation of skeletal muscle primary muscle cell}

In order to perform one experimental procedure for isolating single muscle fiber, C57BL/6 mouse $(\mathrm{N}=3)$ was cervical dislocated and harvested its gastrocnemius. The skeletal muscle was put into a high glucose Eagle's minimal essential medium (DMEM) buffer containing $0.2 \%$ type I collagenase and then it was incubated at 80 90 RPM for two hours in $37^{\circ} \mathrm{C}$ shaking water bath. When enzyme separation was finished, separated muscle single fiber was moved to petri dish Muscle fiber collected by using pasture pipette. Then the muscle fiber moved to new culture dish containing DMEM-FBS buffer that contains $10 \%$ horse serum and $10 \%$ Fatal bovine serum (FBS) and stored in $\mathrm{CO}_{2}$ incubator until the experiment.

\section{Knock down of SIRT1 and GCN5 gene}

The separated muscle cell (500 600 number) move to $100 \mathrm{~mm}$ calture dish and lenti virus particle which contained SIRT1 (SC-40987, santacruz, USA) and GCN5 (SC-37947, Santacruz, USA) shRNA was transfected. We followed the manufacturer's guidelines for virus particle concentration (2 $\mu \mathrm{g} / 5 \mathrm{ml}$ ) and duration (24 hours). Afterwards cells that had not been transfected were removed through puromycin $(5 \mu \mathrm{g} / 5$ $\mathrm{ml})$.

\section{Electrical stimulation on single muscle fiber}

Electrical stimulation on single muscle fiber was conducted based on experiment by Hirano et al. [12]. Skeletal muscle single fiber stored in DMEM medium buffer was moved to Krebs Ringer buffer (115 mM NaCl, $5.9 \mathrm{mM} \mathrm{KCl}, 1.2 \mathrm{mM}$ $\mathrm{MgCl}_{2}, 1.2 \mathrm{mM} \mathrm{NaH}_{2} \mathrm{PO}_{4}, 1.2 \mathrm{mM} \mathrm{Na}_{2} \mathrm{SO} 4,2.5 \mathrm{mM} \mathrm{CaCl}_{2}$, $25 \mathrm{mM} \mathrm{NaHCO}_{3}, 5 \mathrm{mM}$ glucose, $\mathrm{pH}$ 7.4) and then 70 80 muscle fibers were moved to $1.5 \mathrm{ml}$ tube. After stabilizing it in a $37^{\circ} \mathrm{C}$ water tank for five minutes, it was electrically stimulated by an electronic stimulator (DS2A mk2. digitimer. England), using carbon electrode. Electrical stimulation composed to $1 \mathrm{~Hz}$ frequency and three minutes duration. The samples that completed the electrical stimulation was immediately frozen with liquid nitrogen and stored at a $-80^{\circ} \mathrm{C}$ deep freezer until the experiment.

\section{Immunoprecipitation and western blotting}

The frozen sample was lysised in a lysis buffer (Invitrogen, USA) and performed immunoprecipitation according to immunoprecipitation method provided by Abcam. $20 \mathrm{ul}$ of protein G (p7700, Sigma-Aldrich, USA) was added for three hours to preclear immunoglobin and then centrifugated to obtain supernatant at 15000 Rpm for 10 minutes. PGC-1a antibody (1:1000) (AB3242, millipore, USA) and $30 \mathrm{ul}$ of protein $G$ were added to the precleared supernatant then incubated for 12 hours at $4^{\circ} \mathrm{C}$. The protein $\mathrm{G}$ agarose was washed with cold PBS for 15 minutes three times. The immunoprecipitated protein was illusioned and blotted by western blotting method. PGC-1a deacetylation was measured by acetyl-lysine antibody (9441, ABcam, USA). Expression of proteins or acetylation of protein was compared by using 
general western blotting. SIRT1 (07-131, Millipore, USA), GCN5 (sc-365321, Santacruz, USA), AMPK (SC-74461, Santacruz, USA), p-AMPK thr 172 (sc-33524, Santacruz, USA), ACC (SC-30212, Santacruz, USA), p-ACC Ser78/80 (sc-30447, Santacruz, USA) antibody was used for western blotting.

\section{Statistical analysis}

All data are expressed as mean \pm SEM. Statistical analyses were performed using a One-way ANOVA when comparing each groups in the in vitro and in vivo studies. All analyses were performed using SPSS 20.0 (SPSS Inc. USA). Each value is the mean of at least two separate experiments in each group. $\mathrm{P}<0.05$ was considered statistically significant.

\section{RESULTS}

Sirtinol do not inhibited ES induces PGC-1 a deacetylation.

Muscle contraction by electrical stimulation increased expression of SIRT1 $(p<0.05, p=0.004)$ and GCN5 $(p<$ $0.05, p=0.003$ ) level significantly (Fig. $1 \mathrm{~A}$ and D). Sirtinol (100 $\mu \mathrm{M}$, Sigma-aldrich, USA), a SIRT1 inhibitor used for SIRT1 inhibition. Indeed, Sirtinol treated skeletal muscle cell showed significaltly decreased SIRT1 expression level $(p<$ $0.05, p=0.007$ ) (Fig. 1 B), however sirtinol treatment not inhibited PGC-1a deacetylation (Fig. 1 C) $(p=0.294)$. This resulted indicated that SIRT1 inhibition not influenced PGC-1 a deacetylation.

A

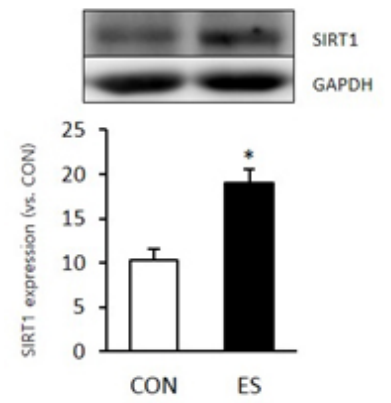

B
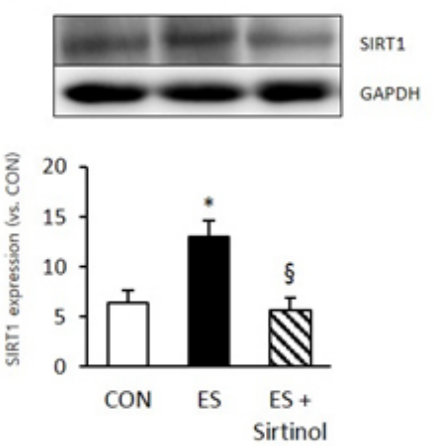

SIRT1 knock down (KD) do not inhibited ES induced $P G C-1$ a deacetylation.

To identify whether SIRT1 genes deleted by SIRT1 lentiviral shRNA treatment, SIRT1 expression level compared with normal cell. SIRT lentiviral shRNA treated cell showed significantly reduced SIRT1 level (Fig. 2A). Next we examined the effect of SIRT KD to other energy metabolsim related protein expression or phosphorylation during muscle contraction. Muscle contraction significantly induced AMPK phosphorylation $(p<0.05, p=0.002)$ compared to non treated cell (wildtype, WT). However SIRT1 KD not influenced to muscle contracition induced p-AMPK level $(p=0.435)$. Muscle contraction also significantly increased phospho-acetyl CoA carboxylase (p-ACC) level compare to WT ( $p<0.05, p=$ 0.001), whereas SIRT1 KD not influenced to muscle contracition induced $\mathrm{p}$-ACC level $(p=0.416)$ (Fig. $2 \mathrm{C}$ ). In immunoprecipitation (IP) results that used PGC-1a antibody, muscle contraction induced Acetyl-lysine level significantly decreased in WT group $(p<0.05, p=0.002)$ however difference between SIRT WT and SIRT1 KD was not significant ( $p=$ 0.435)(Fig. 2D). This result indicated that SIRT1 KD not influenced to muscle contraction induced PGC-1a deacetytilation and muscle energy metabolism.

\section{GCN5 knock down (KD) inhibited ES induced PGC-1 a deacetylation.}

Due to SIRT1 KD not influenced muscle contraction induced PGC-1a deacetylation, we examined other acetly transfrease GCN5 KD effect to PGC-1a deacetylation and energy metabosim related protein expression and phosphorylation. To identify whether GCN5 gene delated by GCN5 lentiviral

C
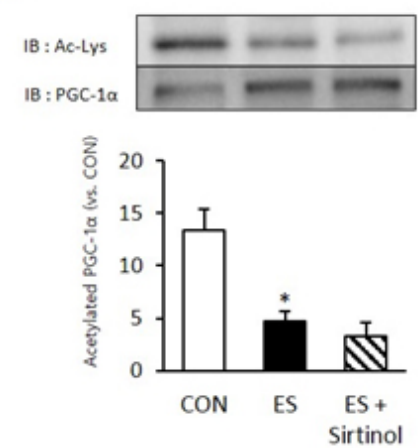

D
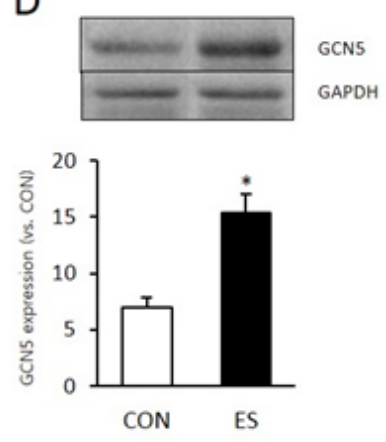

Fig. 1. Sirtinol do not inhibited ES induces PGC-1a deacetylation. A : Electrical stimulation increased SIRT1 expression in skeletal muscle cell. B : 15 min of sirtinol $(100 \mathrm{uM})$ treatment impaired 3 minute of electrical stimulation induced SIRT1 expression. C : 15min of Sirtinol (100uM) not influenced to 3 minute of electrical stimulation induced PGC-1a expression and acetylation. D : Electrical stimulation increased GCN5 expression In skeletal muscle cell. PGC-1a acetylation was measured by PGC-1a immunoprecipitation (IP) with Acetyl-lysine(Ac-Lys). * $p<.05$ between con and ES. ${ }^{\S} p<.05$ between ES and sirtinol treatment. $P$ values were calculated by t-test 
A

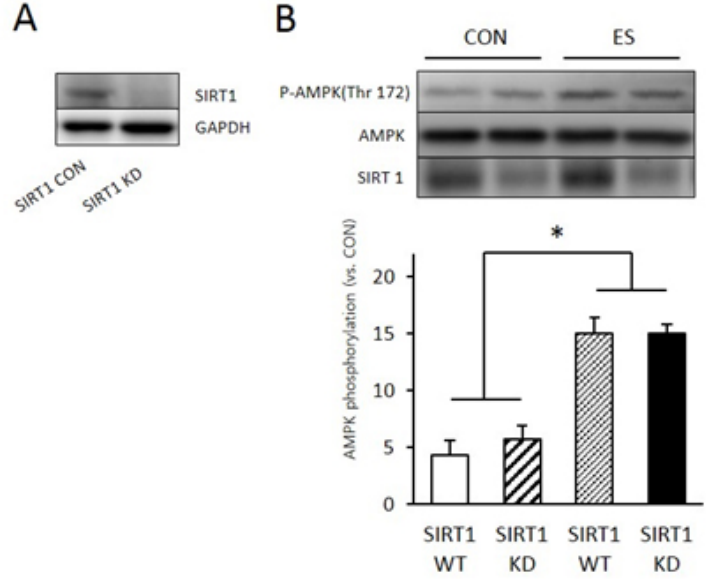

C
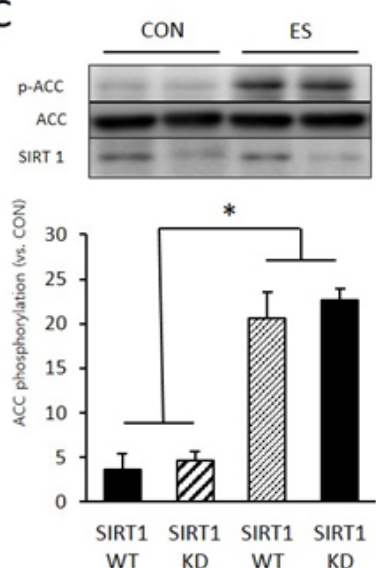
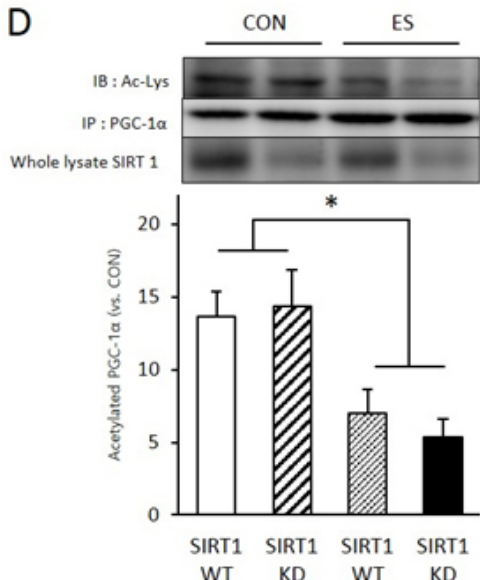

Fig. 2. SIRT1 knock down (KD) do not inhibited ES induced PGC-1a deacetylation. A: Sirt1 SH RNA treatment (knock down, KD) reduced SIRT1 protein level in skeletal muscle cell. B: 3 minute of electrical stimulation induced p-AMPK expression in SIRT 1 shRNA non treat muscle cell (WT) and SIRT KD muscle cell. C: 3 minute of electrical stimulation induced p-ACC expression in SIRT 1 shRNA non treat muscle cell (WT) and SIRT KD muscle cell. D: 3 minute of electrical stimulation induced acetylation of PGC-1a in SIRT 1 shRNA non treat muscle cell (WT) and SIRT KD muscle cell. PGC-1a acetylation was measured by PGC-1a immunoprecipitation (IP) with Acetyl-lysine (Ac-Lys). ${ }^{*} p<.05$ between CON and ES. $P$ values were calculated by one-way ANOVA

A

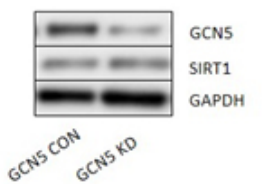

B

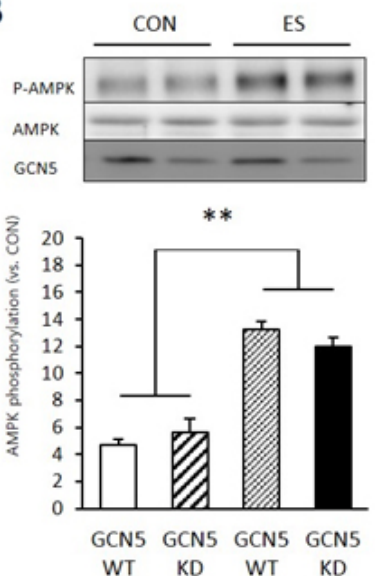

C

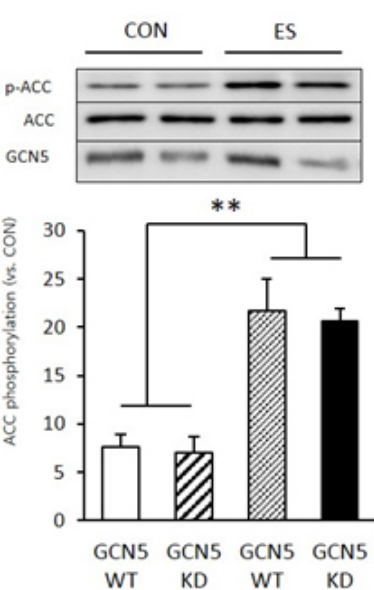

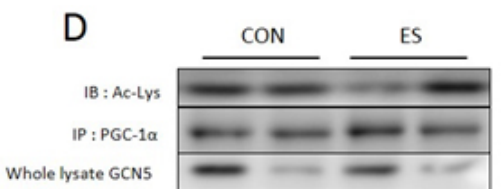

Whole lysate GCNS

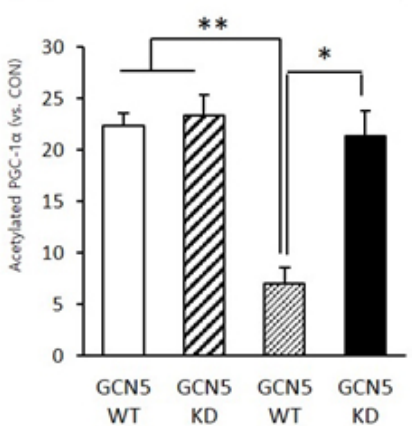

Fig. 3. GCN5 knock down (KD) inhibited ES induced PGC-1á deacetylation. A: GCN5 shRNA treatment (knock down, KD) reduced GCN5 protein level in skeletal muscle cell. B: 3 minute of electrical stimulation induced p-AMPK expression in GCN5 RhRNA non treat muscle cell (WT) and GCN5 KD muscle cell. C: 3 minute of electrical stimulation induced p-ACC expression in GCN5 shRNA non treat muscle cell (WT) and GCN5 KD muscle cell. D: 3 minute of electrical stimulation induced acetylation of PGC-1a in GCN5 shRNA non treat muscle cell (WT) and GCN5 KD muscle cell. PGC-1a acetylation was measured by PGC-1á immunoprecipitation(IP) with Acetyl-lysine(Ac-Lys). ${ }^{*} p<.05$ between WT and KD. ${ }^{* *} p<.05$ between CON and ES. $P$ values were calculated by one-way ANOVA

shRNA on skeletal muscle cell, GCN5 lentiviral lentiviral treated cell showed significantly reduced GCN5 level (Fig. 3 A). Next we examined the effect of GCN5 KD to other energy metabolsim related protein expression or phosphorylation during muscle contraction. Muscle contraction significantly induced AMPK phosphorylation $(p<0.05, p=$ 0.001) compared to non treated cell (wildtype, WT). However SIRT1 KD not influenced to muscle contracition induced p-AMPK level $(p=0.105)$ (Fig. 2A). Muscle contraction also significantly increased phospho-acetyl CoA carboxylase
(p-ACC) level compare to WT ( $p<0.05, p=0.004)$, whereas SIRT1 KD not influenced to muscle contracition induced p-ACC level $(p=0.708)$ (Fig. 3C). In immunoprecipitation (IP) results that used PGC-1a antibody, muscle contraction induced Acetyl-lysine level significantly decreased in WT group ( $p<0.05, p=0.001)$ however difference GCN5 KD cell acetyl-lysine level not significanlty decreased by muscle contraction ( $p=0.735)$ (Fig. 2D). This resulted indicated that GCN5 is important factor for muscle contraction induced PGC-1a deacetylation. 


\section{DISCUSSION}

Skeletal muscle contraction changes intracellular NAD/ NADH levels and energy metabolism through activating energy metabolism related enzymes upregulation and gene transcription [13,14]. SIRT1 and PGC-1a are important factors that regulate non-exercise activity thermogenesis during and after exercise. These protein are closely related to metabolic disorders such as obesity and type 2 diabetes [15]. In this study, we showed SIRT1 protein KD failed to inhibit muscle contraction induced PGC-1a deacetylation however GCN5 KD significantly decreased muscle contraction induced PGC-1a deacetylation.

Skeletal muscle contraction increases $\mathrm{NAD}^{+}$metabolism by activating phosphorylation of AMPK and salvage mechanism of NAMPT. SIRT1, a NAD-dependent protein deacetylase, is controlled by NAD/NADH levels and plays an important role in deacetylation of PGC-1a, a key transcription coactivator regulating energy metabolism. Decreased SIRT1 activity by type 1 diabetes results in inhibition of PGC-1a [16]. However, some recent studies reported that there can be diverse mechanisms for PGC-1a deacetylation and activation because some results indicated that SIRT1 KD or overexpression failed to regulate activation of PGC-1a. [7,17].

In this study, phosphorylation of AMPK and ACC level was measured for assumed energy metabolism during muscle contraction. SIRT1 and GCN5 gene knock down for examine whether gene KD influenced to PGC-1a deacetylation. Skeletal muscle contraction activates cell energy metabolism and increases p-AMPK and p-ACC [6]. However, as seen in the Fig. 2, SIRT1 gene KD failed to inhibit phosphorylation of AMPK and ACC and deacetylation of PGC-1a during skeletal muscle contraction. This indicates that not only SIRT1 but also other histone deacetylases are involved in mechanism of PGC-1a deacetylation. Moreover it indicated that phospholation of AMPK and ACC regulated by other mechanism.

Skeletal contraction increases expression of PGC-1a by facilitating mitochondrial metabolism and it also activates peroxisome proliferator-activated receptor (PPAR) gene transcription to facilitate energy metabolism [18]. In this study, GCN5 KD significantly inhibited muscle contraction induced PGC-1a deacetylation (Fig. 3). Unlike SIRT1, GCN5 as an acetyltransferase is known to regulate PGC-1a deacetylation [10] but its exact mechanism in skeletal muscle has not been clear. Although it has been known that overexpression of GCN5 inhibited expression of PGC-1a deacetylation [19], a few studies reported the mechanism of GCN5 KD activation and PGC-1a acetylation during muscle contraction. However, it is known that GCN5 stimulates PGC-1a serine 570 phos- phorylation through angiotension II mechanism and controls PGC-1a lysine acetylation, indicating that AKT-dependent PGC-1a signaling system [20]. Intriguingly, several studies have suggested that interaction between SIRT1 and other SIRT isotypes controls PGC-1a acetylation. While SIRT1 stimulates PGC-1a deacetylation, SIRT6 controls PGC-1a acetylation [10]. AMPK and protein kinase A (PKA) which are activated during exercise inhibit too much energy production, GCN5 activation by SIRT6 controls the level of PGC-1a acetylaiton [21]. These results indicate that GCN5 activation can be controlled by multiful factors such as phosphoinisitide 3 kinase (PI3K), AKT, p38 mitogen activated protein kinases that can be increased by exercise. Additional studies to these factor by using inhibitor experiments of relevant factors or gene knockdown may be needed. Also when PGC-1a is transferred into a cell nucleus, it unites not only with GCN5, SIRT1 but also with other co-activators such as steroid receptor coactivator-1 (SRC-1) or p300 (Coste et al., 2008), which require additional studies.

\section{CONCLUSION}

This study aimed to identify the factors that control PGC-1a deacetylation during skeletal muscle contraction. To examine the effect of SIRT1 and GCN5 gene KD, lentiviral shRNA transfected to skeletal muscle primay cell and $\mathrm{KD}$ cell were contracted by electrical stimulation. The results showed that SIRT KD did not affect the muscle contraction induced AMPK and ACC phosphorylation, also SIRT KD did not significantly affected the muscle contraction induced PGC-1a deacetylation. Besides GCN5 KD significantly reduced muscle contraction induced PGC-1a deacetylation however it did not affect AMPK and ACC phosphorylation compared to those of control group. In conclusion, these results indicated that GCN5 play an important role in muscle contraction induced PGC-1a deacetylation however further research will be need to identify its exact regulation mechanism.

\section{ACKNOWLEDGEMENTS}

This work was supported by the National Research Foundation of Korea Grant funded by the Korean Government (NRF-2011-354-G00092). 


\section{REFERENCES}

[1] Luo X, Kraus WL. A one and a two $\cdots$ expanding roles for poly (ADP-ribose) polymerases in metabolism. Cell Metab. 2011;6;13(4):353-5.

[2] Cantó C, Jiang LQ, Deshmukh AS, Mataki C, Coste A, Lagouge M, Zierath JR, Auwerx J. Interdependence of AMPK and SIRT1 for metabolic adaptation to fasting and exercise in skeletal muscle. Cell Metab. 2010;3; 11(3):213-9.

[3] Aksoy P, Escande C, White TA, Thompson M, Soares $\mathrm{S}$, Benech JC, Chini EN. Regulation of SIRT 1 mediated NAD dependent deacetylation: a novel role for the multifunctional enzyme CD38. Biochem Biophys Res Commun. 2006;13;349(1):353-9.

[4] Fulco M, Sartorelli V. Comparing and contrasting the roles of AMPK and SIRT1 in metabolic tissues. Cell Cycle. 2008;7(23):3669-79.

[5] Bai P, Cantó C, Oudart H, Brunyánszki A, Cen Y, Thomas C, Yamamoto H, Huber A, Kiss B, Houtkooper RH, Schoonjans K, Schreiber V, Sauve AA, Menissierde Murcia J, Auwerx J. PARP-1 inhibition increases mitochondrial metabolism through SIRT1 activation. Cell Metab. 2011;6;13(4):461-8

[6] Philp A, Chen A, Lan D, Meyer GA, Murphy AN, Knapp AE, Olfert IM, McCurdy CE, Marcotte GR, Hogan MC, Baar K, Schenk S. Sirtuin 1 (SIRT1) deacetylase activity is not required for mitochondrial biogenesis or peroxisome proliferator-activated receptorgamma coactivator-1alpha (PGC-1alpha) deacetylation following endurance exercise. J Biol Chem. 2011;2;286 (35):30561-70.

[7] Gurd, B. J., Perry, C. G., Heigenhauser, G. J., Spriet, L. L., \& Bonen, A. (2010). High-intensity interval training increases SIRT1 activity in human skeletal muscle. Appl Physiol Nutr Metab, 35(3), 350-357.

[8] Puigserver P, Wu Z, Park CW, Graves R, Wright M, Spiegelman BM. A cold-inducible coactivator of nuclear receptors linked to adaptive thermogenesis. Cell. 1998; 92:829-839.

[9] Cantó, C., Gerhart-Hines, Z., Feige, J. N., Lagouge, M., Noriega, L., Milne, J. C., Elliott, P. J., Puigserver, P., \& Auwerx, J. AMPK egulates energy expenditure by modulating $\mathrm{NAD}+$ metabolism and SIRT1 activity. Nature, 2009;23;458(7241), 1056-1060.

[10] Dominy JE Jr, Lee rY, Gerhart-Hines Z, Puigserver $\mathrm{P}(2010)$. Nutrient-dependent regulation of PGC-1alpha's acetylation state and metabolic function through the enzymatic activities of Sirt1/GCN5. Biochim Biophys Acta.
1804(8):1676-83.

[11] Gerhart-Hines Z, Dominy JE Jr, Blättler SM, Jedrychowski MP, Banks AS, Lim JH, Chim H, Gygi SP, Puigserver P. The cAMP/PKA pathway rapidly activates SIRT1 to promote fatty acid oxidation independently of changes in $\mathrm{NAD}(+)$. Mol Cell. 2011;23;44(6):851-63.

[12] Hirano H, Takahashi E, Doi K, Watanabe Y. Role of intracellular calcium in fatigue in single skeletal muscle fiber isolated from thr rat. Patho Physio. 2000;6:211-216

[13] Koltai, E., Szabo, Z., Atalay, M., Boldogh, I., Naito, H., Goto, S., Nyakas, C., \& Radak, Z. (2010). Exercise alters SIRT1, SIRT6, NAD and NAMPT levels in skeletal muscle of aged rats. Mech Ageing Dev, 131(1), 21-28.

[14] Hayes DJ, Byrne E, Shoubridge EA, Morgan-Hughes JA, Clark JB(1985). Experimentally induced defects of mitochondrial metabolism in rat skeletal muscle. Biological effects of the NADH: coenzyme Q reductase inhibitor diphenyleneiodonium. Biochem J 229(1) : 109-17

[15] Ha TG, Kim JC. The Effects of Endurance Training Combined with Rosiglitazone on The Expression of PPARs, PGC-1a, GLUT-4 and p-AMPK-a2 in The Skeletal Muscle of Diabetic Induced-Rats. Journal of exercise nutrition \& biochemistry. 2009; 13(2). 131-140.

[16] Li L, Pan R, Li R, Niemann B, Aurich AC, Chen Y, Rohrbach S. Mitochondrial biogenesis and peroxisome proliferator-activated receptor-y coactivator-1a (PGC-1a) deacetylation by physical activity: intact adipocytokine signaling is required. Diabetes. 2011;60(1):157-67.

[17] Boily G, Seifert EL, Bevilacqua L, He XH, Sabourin G, Estey C, Moffat C, Crawford S, Saliba S, Jardine K, Xuan J, Evans M, Harper ME, McBurney MW. SirT1 regulates energy metabolism and response to caloric restriction in mice. PLoS One. 2008;12;3(3):e1759.

[18] Jo JS, Jand HS, Yoon JS, Kim JC. Endurance Traininginduced Expressions of PPAR-a, PPAR- 8 and PGC-1a Protein in Skeletal Muscles of Rats Journal of exercise nutrition \& biochemistry. 2006;10(3). 289-296.

[19] Lerin C, Rodgers JT, Kalume DE, Kim SH, Pandey A, Puigserver P. GCN5 acetyltransferase complex controls glucose metabolism through transcriptional repression of PGC-1alpha. Cell Metab. 2006;3(6):429-38.

[20] Xiong S, Salazar G, San Martin A, Ahmad M, Patrushev N, Hilenski L, Nazarewicz RR, Ma M, Ushio-Fukai M, Alexander RW. PGC-1 alpha seriGCN5-mediated acetylation by angiotensin II drive catalase down-regulation and vascular hypertrophy. J Biol Chem. 2010;285(4): 2474-87.

[21] Jeninga EH, Schoonjans K, Auwerx J. Reversible acetylation of PGC-1: connecting energy sensors and effectors 
to guarantee metabolic flexibility. Oncogene. 2010; 19;29(33):4617-24.

[22] Coste A, Louet JF, Lagouge M, Lerin C, Antal MC, Meziane H, Schoonjans K, Puigserver P, O'Malley BW,
Auwerx J. The genetic ablation of SRC-3 protects against obesity and improves insulin sensitivity by reducing the acetylation of PGC-1alpha. Proc Natl Acad Sci U S A. 2008;105(44):17187-92. 